\title{
A GLP-2 Analogue Protects SH-SY5Y and Neuro-2a Cells Against Mitochondrial Damage, Autophagy Impairments and Apoptosis in a Parkinson Model
}

\author{
Authors

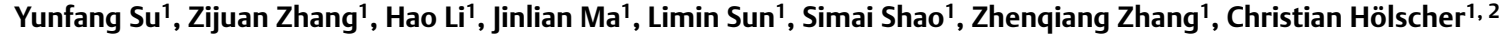

\begin{abstract}
Affiliations
1 Academy of Chinese Medical Sciences, Henan University of Chinese Medicine, Zhengzhou, Henan, China

2 Neurology Department of the Second Associated Hospital of Shanxi Medical University, Taiyuan, Shanxi, China
\end{abstract}

Key words

Biologics, central nervous system disorders, neuropharmacology, neuropeptides, growth factors / neurotrophic factors

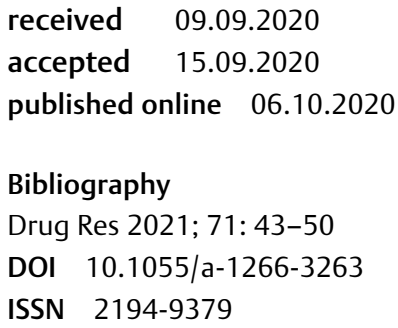

\section{Correspondence}

Prof. Christian Hölscher

Academy of Chinese Medical Sciences, Henan University of Chinese Medicine,

Zhengzhou 450046,

Henan Province,

China

Tel.: 18339962156

c.holscher@hactcm.edu.cn

\begin{abstract}
Glucagon-like peptide-2 (GLP-2) is a peptide hormone that belongs to the glucagon-derived peptide family. We have previously shown that analogues of the sister hormone Glucagonlike peptide-1 (GLP-1) showed neuroprotective effects. Here we investigated the effect of a GLP-2 agonist in a cell model of Parkinson's disease (PD) created by treating SH-SY5Y or Neuro2 a cells with 1-Methyl-4-phenyl-pyridine ion $(\mathrm{MPP}+)$. Cell viability and cell cytotoxicity was detected by MTT and LDH assays, respectively. The protein expression levels of mitochondrial, autophagy and apoptotic biomarkers including PGC-1 $\alpha$, Mfn2, IRE1, ATG7, LC3B, Beclin 1 and Bcl-2 were detected by western blot. Mitochondrial superoxide was detected by MitoSOX Red. In addition, mitochondrial morphology, autophagosome and apoptotic corpuscles were observed by transmission electron microscope (TEM). We found that the GLP-1 and the GLP-2 agonists both protect cells against mitochondrial damage, autophagy impairments and apoptosis induced by MPP + both in SH-SY5Y and Neuro-2a cells. Cell signaling for mitogenesis was enhanced, and oxidative stress levels much reduced by the drugs. This demonstrates for the first time the neuroprotective effects of a GLP-2 analogue in PD cellular models, in which oxidative stress, autophagy and apoptosis play crucial roles. The protective effects were comparable to those seen with the GLP-1 analogue liraglutide. The results suggest that not only GLP-1, but also GLP-2 has neuroprotective properties and may be useful as a novel treatment of PD.
\end{abstract}

\section{Introduction}

Glucagon-like peptide-2 (GLP-2) is a hormone with 33-amino acid derived from proglucagon in the gut and central nervous system (CNS). In 2012, the first GLP-2 peptide analogue, teduglutide, was approved for the treatment of short bowel syndrome (SBS) and following this, an increasing number of GLP-2 peptide analogues were developed for clinical use [1]. GLP-2 is a hormone that regulates food intake [2], inhibits growth of osteosarcoma cells and promotes directed differentiation of osteoblasts [3], and furthermore shows cytoprotective effects in combat lung injury [4]. However, the role of GLP-2 in the CNS had only recently been studied. It has been reported that GLP-2 has protective effects in a mouse model of sporadic Alzheimer's disease (AD) in which streptozotocin is injected directly into the brain to induce neurodegenerative processes and insulin desensitization [5], and that the drug reduces obesity-related neuroinflammation [6]. It furthermore is neuroprotective in a cerebral ischemia/reperfusion model of stroke [7], in an animal model of vascular dementia [8], chronic cerebral hypoperfusion [9] and in several mouse models of depression [10-12].

GLP-2 is synthetized in selected tissues by cleaving proglucagon [13]. Proglucagon is expressed tissue-specifically and is cleaved into 
GLP-1, GLP-2, and glicentin in the intestine and brain by the hormone proto-invertase. In the brain, GLP-2 is found in discrete clusters of neurons in the brain stem and hypothalamus [14]. GLP-2 receptor (GLP-2R) expressing neurons have been identified in the Cortex, the hippocampus, the ventromedial hypothalamic nucleus, the nucleus of the solitary tract, the parabrachial nucleus, the supramammillary nucleus, and the substantia nigra. Moreover, GLP-2 showed cytoprotective properties in cells derived from the CNS [15].

The biological activity of GLP-2 is mediated by GLP-2R, a member of the G-protein coupled receptor superfamily. GLP-2R is highly expressed in the intestinal tract [16] and exists in the CNS also $[2,15,17,18]$. In mouse hippocampal and cortical cells, GLP-2 stimulates CAMP production and reduces glutamate-induced neuronal death and cell death induced by apoptosis [15]. Similarly in mouse hippocampal neurons, GLP-2 activated mouse hippocampal neurons through PKA signaling, and increased glucose uptake in a PKAdependent manner [19]. In addition to the CAMP pathway, the GLP-2 receptor activates the PLC-PI3k-Akt-mTOR pathway which is the same second- messenger pathway that the insulin receptor activates [20]. A new study suggested that GLP2R and the AKTmTOR-p70S6K pathway in the hippocampus are promising targets to treat cognition deficits in a chronic cerebral hypoperfusion animal model [9]. Another pathway is ERK signaling through which GLP-2 can regulate cognitive function [8]. It appears that GLP-2R signaling pathways act in energy utilization, block apoptosis, induce insulin re-sensitization, enhance protein synthesis, reduce inflammation and glia activation, and enhance cell repair and growth.

We have previously shown that activating the GLP-1 pathway has neuroprotective effects in animal models of Alzheimer's and Parkinson's disease [21-23]. A phase II clinical trial in PD patients showed that a GLP-1 receptor agonist is effective in treating PD patients [24]. GLP-1 is co-released with GLP-2 during the processing of proglucagon [25]. However, the protective effects and the mechanisms of GLP-2 signaling in models of Parkinson `s disease (PD) have not been investigated. PD is the second major neurodegenerative disease in the world that no drugs can stop or even reverse disease progression in it. In this study, we investigated the effect of a protease-resistant GLP-2 analogue in cell models of PD induced by 1-Methyl-4-phenyl-pyridine ion (MPP + ) which is the active metabolite of 1-methyl-4-phenyl-1,2,3,6-tetrahydropyridine (MPTP). We tested if the GLP-2 agonist restores mitochondrial damage, autophagy impairments and apoptotic in SH-SY5Y and Neuro-2a cells.

\section{Materials and Methods}

\section{Materials}

Lactate dehydrogenase (LDH) kit was purchased from Roche Diagnostics Ltd (West Sussex, UK). PGC-1 $\alpha$ antibody (ab54481), Mitofu$\sin 2$ antibody (ab124773), IRE1 antibody (ab48187), ATG7 antibody (ab133528), LC3B antibody (ab48394), Beclin1 antibody (ab207612) and ECL Western Blotting Substrate were purchased from Abcam (MA, USA). Bcl-2 antibody and horseradish peroxidase (HRP)-linked secondary antibodies against rabbit and mouse IgG of the primary antibodies were from BOSTER (Wuhan, China). Bovine serum albumin (BSA) and MPP + were obtained from Sigma-Aldrich Company Ltd (Dorset, UK). DMEM/F-12 and fetal bovine serum (FBS) were pur- chased from Gibco. DMEM high glucose was from Biological Industries Ltd (HaZafon, Israel). Penicillin-Streptomycin solution, $0.25 \%$ trypsin-EDTA solution without phenol red, NP-40 lysis buffer, MTT, BCA protein concentration determination kit, paraformaldehyde and dimethyl sulfoxide (DMSO), phosphate buffered saline (PBS; $\mathrm{pH} 7.4$ ), Tween 20, $5 \times$ SDS-PAGE sample loading buffer and other reagents for cell culture and western blotting were obtained from Solarbio (Beijing, China). MitoSOX Red mitochondrial superoxide indicator (M36008) was purchased from Invitrogen. The peptides of GLP-2 agonist and Liraglutide were synthesized by China Peptides (Shanghai, China), the amino acid sequence of the GLP-2 analogue is HGDGSFSDEMSTILDNLATRDFINWLIQTKITD. The amino acid sequence of Liraglutide is HAEGTFTSDVSSYLEGQAAK[( $\gamma E)-($ Pal)]EFIAWLVRGRG; (Pal = palmitoyl acid). The purity of the peptides is $95 \%$.

\section{Cell culture}

The human neuroblastoma SH-SY5Y cell line (ATCC ${ }^{\circledR}$ CRL-2266) and Neuro-2a cell line (ATCC ${ }^{\circledR}$ CCL-131) were obtained from Cell Resource Center, Shanghai Institute of life sciences, Chinese Academy of Sciences (Shanghai, China). SH-SY5Y cells were cultivated in Dulbecco's modified eagle medium/nutrient mixture F-12 (DMEM/F-12, $1: 1 ; 1 \mathrm{X})$ Glutamax supplemented with $10 \% \mathrm{FBS}, 100 \mathrm{IU} \mathrm{ml}^{-1}$ of Penicillin and $100 \mu \mathrm{g} \cdot \mathrm{ml}^{-1}$ of Streptomycin. Neuro-2a Cells were cultivated in Dulbecco's modified eagle medium (DMEM, $1 \times$, high glucose) Glutamax supplemented with $10 \% \mathrm{FBS}, 100 \mathrm{IU} \mathrm{ml}^{-1}$ of Penicillin and $100 \mu \mathrm{g} \cdot \mathrm{ml}^{-1}$ of Streptomycin. Cells were maintained at $37^{\circ} \mathrm{C}$ in a humidified incubator with $5 \% \mathrm{CO}_{2}$ and $95 \%$ air. Cells were subcultured when $80-90 \%$ confluent and seeded at 1:4 ratio. Culture medium was renewed every 3 to 4 days.

\section{Cell treatment}

$\mathrm{MPP}+$ was diluted in PBS at a concentration of $100 \mathrm{mM}$, aliquoted and stored at $-20^{\circ} \mathrm{C}$ until used. GLP-2 agonist and Liraglutide were diluted in sterile water at a concentration of $1 \mathrm{mM}$, aliquoted and stored at $-20^{\circ} \mathrm{C}$ until used as well. For the experiments, firstly, $\mathrm{MPP}+$ was diluted in culture medium at concentrations of $0.5 \mathrm{mM}$, $1 \mathrm{mM}, 2 \mathrm{mM}$ and $4 \mathrm{mM}$, respectively, to confirm a lowest concentration that decreased the cell viability significantly and to be applied in subsequent experiments. GLP-2 agonist was diluted in culture medium at concentrations of $1 \mathrm{nM}, 10 \mathrm{nM}, 100 \mathrm{nM}$ and $200 \mathrm{nM}$, to select a concentration that reversed the cell damage significantly and to be applied in subsequent experiments. Liraglutide stock preparations were diluted in culture medium to a final working concentration of $100 \mathrm{nM}$ which was selected on the basis of previous experiments [26-28].

\section{Cell viability and cytotoxicity assessment}

Cell viability and cytotoxicity were determined using MTT and LDH assay, respectively. The assays were performed in 96-well plates. Both of SH-SY5Y cells and Neuro-2a cells were seeded at a density of $3 \times 10^{4}$ cells in $100 \mu \mathrm{L}$ per well for $24 \mathrm{~h}$ for use. In order to confirm the concentration of MPP + for the cell model of PD, the cells were stressed with different concentrations of MPP + $(0.5 \mathrm{mM}, 1 \mathrm{mM}$, $2 \mathrm{mM}$ and $4 \mathrm{mM}$ ) for $16 \mathrm{~h}$, then the cell viability and cytotoxicity were determined. For the drug test, cells were pretreated with $0 \mathrm{nM}, 1 \mathrm{nM}, 10 \mathrm{nM}, 100 \mathrm{nM}$ and $200 \mathrm{nM}$ of GLP-2 agonist for $12 \mathrm{~h}$, respectively, MPP + was added subsequently for another $16 \mathrm{~h}$, a 
concentration that reversed the cell damage significantly was determined. Following cell treatments, $50 \mu \mathrm{L}$ of the supernatant was collected and the cell cytotoxicity was assessed according to the manufacturer's instructions: the collected cell supernatant was incubated with the reaction mixture for $30 \mathrm{~min}$ at room temperature, then the reaction was terminated by adding the stop solution from the kit and absorbance was measured at $490 \mathrm{~nm}$ using a microplate reader. The absorbance values are proportional to the cytotoxicity of the cells damage. Meanwhile, $20 \mu \mathrm{L}$ of MTT $(5 \mathrm{mg} / \mathrm{mL})$ was added to each well and maintained at $37^{\circ} \mathrm{C}$ in a humidified incubator, after incubating for $4 \mathrm{~h}$, the supernatants were removed and $100 \mu \mathrm{L}$ of DMSO was added to each well. After the formazan crystals dissolved completely, the absorbance was measured at $570 \mathrm{~nm}$ using a microplate reader. The absorbance values are proportional to the number of viable cells. All cell treatments were performed in sextuplicate per plate per experiment.

\section{Western blotting}

$2 \times 10^{6}$ cells were seeded at $6 \mathrm{~cm}^{2}$ dish for $24 \mathrm{~h}$. Cells were pretreated with $100 \mathrm{nM}$ of GLP-2 agonist or Liraglutide for $12 \mathrm{~h}$ before stressed with $2 \mathrm{mM}$ of MPP + for another $16 \mathrm{~h}$. Thereafter, cells were washed once with ice-cold $1 \times \mathrm{PBS}$ and harvested by $0.25 \%$ trypsin and centrifugation at $1000 \mathrm{rpm}$ at $4{ }^{\circ} \mathrm{C}$ for $5 \mathrm{~min}$. Cells were resuspended in $1 \times$ cell lysis buffer containing protease/phosphatase inhibitor $(1 \times)$ and kept on ice for $30 \mathrm{~min}$. Total protein was extracted by centrifugation at $12000 \mathrm{rpm}$ at $4^{\circ} \mathrm{C}$ for $10 \mathrm{~min}$. BCA protein concentration determination kit was conducted to estimate the protein concentration of the samples, according to the manufacturer's instructions. Protein in lysate $(10 \mu \mathrm{g})$ was boiled with $5 \times$ SDS-PAGE sample loading buffer at $99^{\circ} \mathrm{C}$ for $10 \mathrm{~min}$ to be denatured. Protein samples were separated on $10 \%$ gradient Bis-Tris gel and blotted onto nitrocellulose membranes using Bio-Rad System. Blots were blocked in $5 \%(\mathrm{w} / \mathrm{v})$ skimmed milk in $1 \times$ PBS with $0.05 \%$ Tween 20 (PBST) for $1 \mathrm{~h}$ at room temperature, and the blots were cut apart based on the protein molecular weight and incubated with the primary antibodies against PGC- $1 \alpha(1: 2000)$, Mfn2 (1:1000), IRE1 (1:2500), ATG7 (1:10000), LC3B (1:1000), Beclin 1 (1:2000), Bcl-2 $(1: 300)$ and $\beta$-actin (1:400) in $5 \%(w / v)$ skimmed milk overnight at $4{ }^{\circ} \mathrm{C}$. Blots were washed three times in PBST for 10 min each and incubated with the HRP-linked secondary antibodies against the corresponding species $\lg \mathrm{G}(1: 5000)$ for $1 \mathrm{~h}$ at room temperature. Blots were developed using ECL western blotting detection reagent kit as per manufacturer's instructions. Bio-Rad Imaging System was used to image chemiluminescent bands. Image J and Prism 7 software programs were used to perform the densitometric analysis. $\beta$-Actin protein was served as loading control, to which relative peak intensities of the examined proteins were normalized.

\section{Mitochondrial superoxide detection}

$4.2 \times 10^{5}$ cells were seeded at 12 -well plate for $24 \mathrm{~h}$. Cells were pretreated with $100 \mathrm{nM}$ of GLP-2 agonist or Liraglutide for $12 \mathrm{~h}$ before stressed with $2 \mathrm{mM}$ of MPP + for another $16 \mathrm{~h}$. MitoSOX Red mitochondrial superoxide indicator was performed to detect the mitochondrial superoxide. $5 \mu \mathrm{M}$ of MitoSOX reagent working solution diluted in cell culture medium was applied to cover cells. Cells were incubated at $37^{\circ} \mathrm{C}$ for $10 \mathrm{~min}$, protected from light. Cells were washed gently for three times with warm culture medium. Cells were stained with $100 \mu \mathrm{g} / \mathrm{mL} 4^{\prime}$,6-diamidino-2-phenylindole (DAPI) which was diluted in cell culture medium at $37^{\circ} \mathrm{C}$ for $20 \mathrm{~min}$. After washing three times with warm culture medium, cells in culture medium were observed using fluorescence microscope. Images were taken and analyzed using the Image J graphic analysis program (freeware form the NIH, USA). Dead cells were not counted in the quantification of fluorescence. Results were statistically analyzed using the program Prism 7 (GraphPad software, USA).

\section{Transmission electron microscope (TEM)}

To observe mitochondrial morphology, autophagosome and apoptotic corpuscle of the cells in different groups, $2 \times 10^{6}$ cells were seeded in cell culture dish with a diameter of $6 \mathrm{~cm}$ for $24 \mathrm{~h}$. Cells

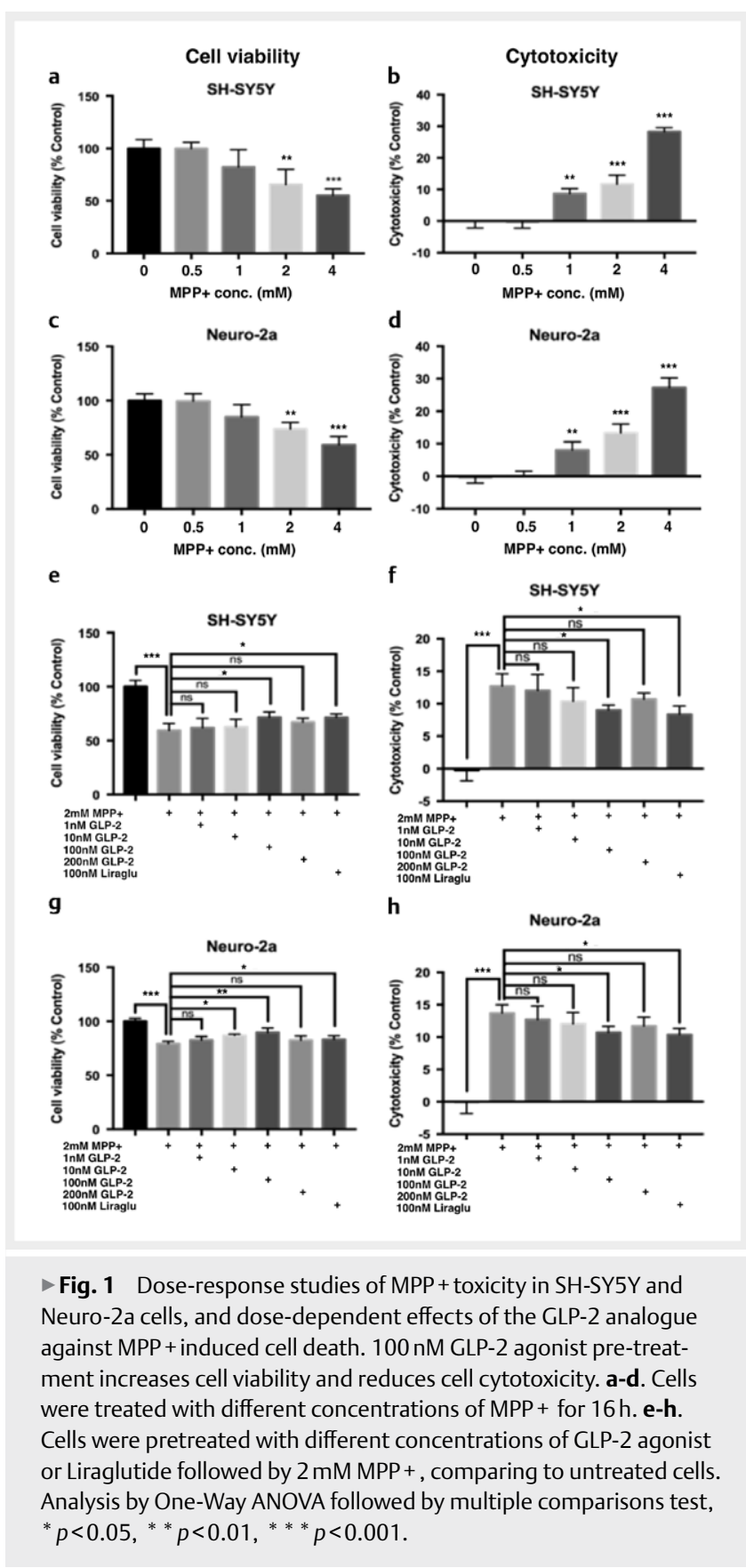




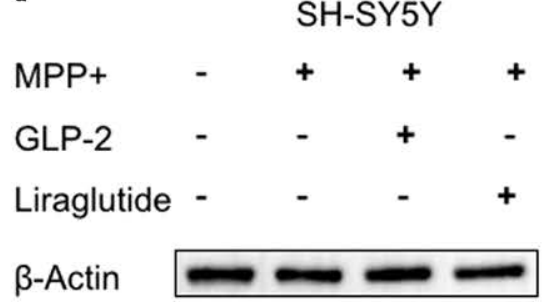

PGC-1 $\alpha$

Mfn2

IRE1

ATG7

$\operatorname{LC3B}(\mathrm{I}, \mathrm{II})$

Bcl-2

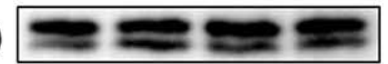

c

$\mathrm{MPP}+$

GLP-2

Liraglutide

$\beta$-Actin

PGC-1a

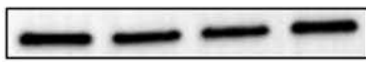

Mfn2

IRE1

ATG7

$\operatorname{LC} 3 \mathrm{~B}(\mathrm{I}, \mathrm{II})$

Bcl-2 b
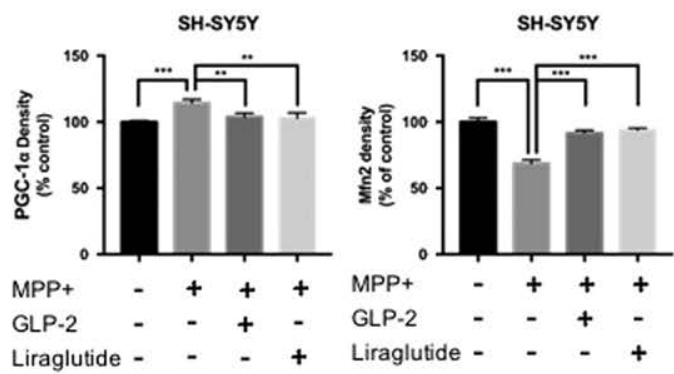

SH-SY5Y

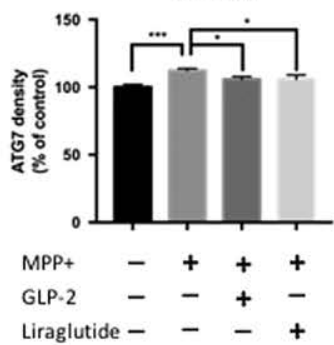

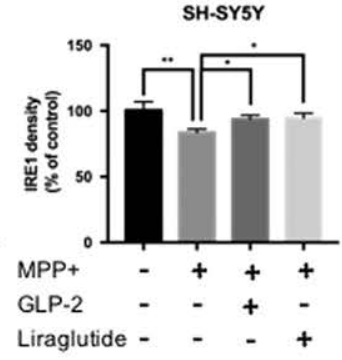

SH-SY5Y

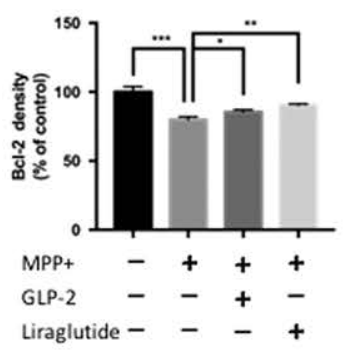

d
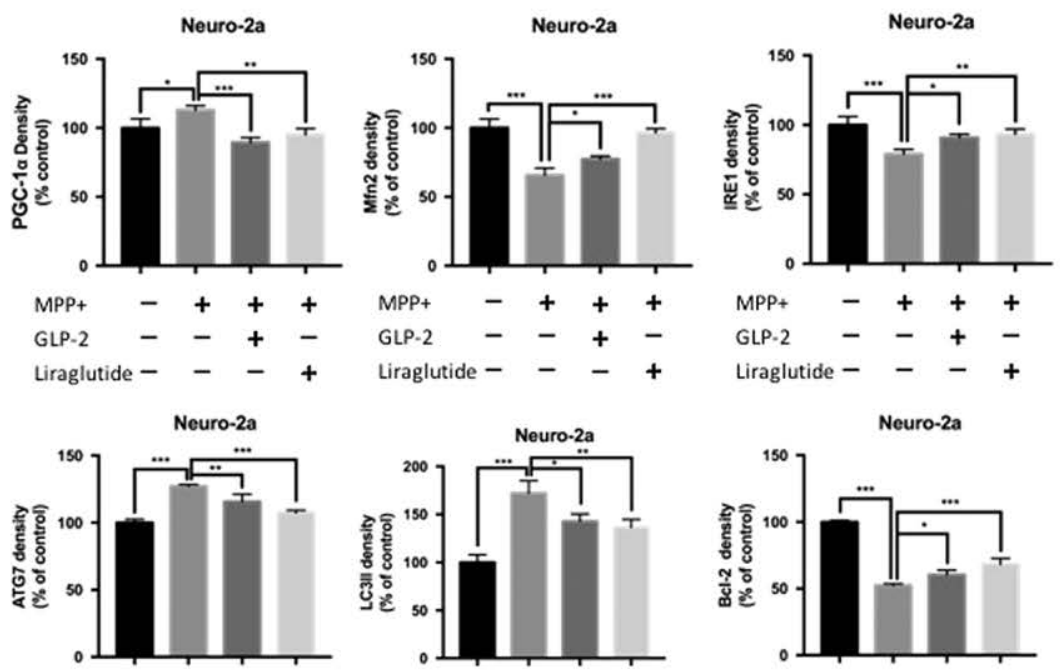

$\mathrm{MPP}_{+}-+++$

GLP-2 - - + -

Liraglutide - $-\quad+$

-Fig. 2 Western Blot analysis of mitochondrial, autophagy and apoptotic biomarkers. a, c. Western blots showing protein expression when cells were pretreated with $100 \mathrm{nM}$ GLP-2 agonist or Liraglutide followed by $2 \mathrm{mM} \mathrm{MPP}+$. b, d. Densitometric analysis. Analysis was performed by One-Way ANOVA followed by multiple comparisons test, ${ }^{*} p<0.05,{ }^{* *} p<0.01,{ }^{* * *} p<0.001$.

were pretreated with $100 \mathrm{nM}$ of GLP-2 agonist or Liraglutide for $12 \mathrm{~h}$ before stressed with $2 \mathrm{mM}$ of MPP + for another $16 \mathrm{~h}$. Each group was performed in triple. Cells were harvested using cell scrapers and centrifugation at $2000 \mathrm{rpm}$ at $4^{\circ} \mathrm{C}$ for $15 \mathrm{~min}$. After fixed with $2.5 \%$ glutaraldehyde for $4 \mathrm{~h}$, the solution was refreshed and rinsed with $0.1 \mathrm{M} \mathrm{PBS}$, then fixed with $1 \%$ osmium acid solution for $2 \mathrm{~h}$, rinsed again with $0.1 \mathrm{M} \mathrm{PBS}$, dehydrated with ethanol and acetone and embedded with epoxy resin 812 finally. After sliced, slices were stained with saturated uranium acetate solution and lead citrate solution. Three slices were observed in each sample after dried by projection electron microscope (JEM-1400, Japan). Mitochondrial morphology, autophagosomes and apoptotic corpuscles were observed. TEM was performed by TEM Center of Henan University of Chinese Medicine.

\section{Statistics}

Statistical analysis in this study was performed using EXCEL and GraphPad Prism 7 software (GraphPad software, USA). The exper- 


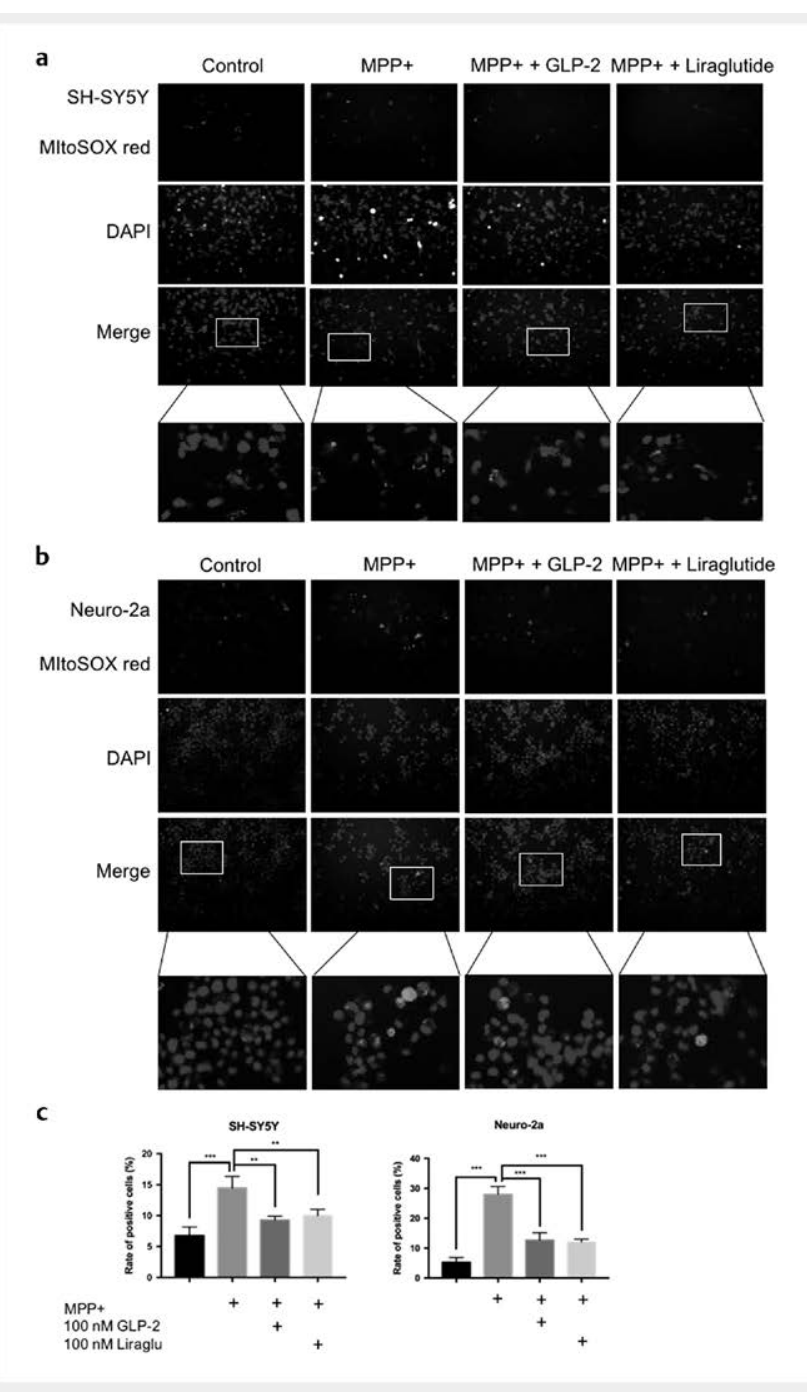

- Fig. 3 Mitochondrial superoxide test using MitoSOX red. a, b showing positive cells for mitochondrial superoxide when cells were pretreated with $100 \mathrm{nM}$ GLP-2 agonist or Liraglutide followed by $2 \mathrm{mM} \mathrm{MPP}+$. c. Positive cell number quantification. Analysis was performed by One-Way ANOVA followed by multiple comparisons test, ${ }^{*} p<0.05,{ }^{* *} p<0.01,{ }^{* * *} p<0.001$.

imental data were analyzed by one-way ANOVA with post-hoc tests or Student's t-tests. Data are shown as Mean \pm SD, and a $P<0.05$ was considered as significant.

\section{Results}

$2 \mathrm{mM}$ MPP + decreases cell viability and increases LDH levels, the GLP-1 and GLP-2 analogues protect cell against damage from MPP + both in SH-SY5Y and Neuro-2a cells

The effective concentration of MPP + for the cell model of PD was estimated in a dose-response test. The cells were stressed with different concentrations of MPP $+(0.5 \mathrm{mM}, 1 \mathrm{mM}, 2 \mathrm{mM}$ and $4 \mathrm{mM})$ for $16 \mathrm{~h}$, then the cell viability and cytotoxicity were determined.
- Fig.1a,c showed at list 2 mM MPP + decreases cell viability significantly comparing to control group in both two cell lines $(P<0.05)$. During the injury of nerve cells, LDH is released into the culture supernatant, and the leakage rate of LDH in the supernatant reflects the degree of cell injury or death. $>$ Fig. $\mathbf{1 b}, \mathbf{d}$ show that at least $1 \mathrm{mM}$ MPP + increases LDH levels significantly comparing to control group in both two cell lines $(P<0.05)$. The cell viability and cytotoxicity had a dose-effect relationship with the concentration of MPP + . Therefore, $2 \mathrm{mM} \mathrm{MPP}+$ was selected to test the drugs subsequently. For the drug test, cells were pretreated with $0 \mathrm{nM}, 1 \mathrm{nM}, 10 \mathrm{nM}, 100 \mathrm{nM}$ and $200 \mathrm{nM}$ of GLP-2 agonist for $12 \mathrm{~h}$, respectively, with $100 \mathrm{nM}$ Liraglutide used as a positive control. MPP + was added subsequently for another $16 \mathrm{~h}$. > Fig. 1e-h showed $100 \mathrm{nM}$ GLP-2 agonist and $100 \mathrm{nM}$ Liraglutide both increased cell survival and reduced cytotoxicity significantly in both two cell lines $(P<0.05)$. GLP-2 agonist alone did not cause a significant change in cell viability and cytotoxicity in both SH-SY5Y and Neuro-2a cells (data not shown).

\section{The drugs protect cells against mitochondrial damage, autophagy impairments and apoptotic signaling induced by MPP+}

PGC- $1 \alpha$ is a key factor that mediates mitochondrial biosynthesis [29]. Mitofusin2 (Mfn2) regulates mitochondrial fusion, transport and autophagy. As shown in > Fig. 2, 2 mM MPP + decreased PGC$1 \alpha$ and Mfn2 levels significantly $(P<0.05), 100 \mathrm{nM} \mathrm{GLP}-2$ agonist and $100 \mathrm{nM}$ Liraglutide both protected cells against the changes of the protein levels induced by MPP + significantly $(P<0.05)$. IRE1 is the core receptor of ER stress, which can sense the accumulation of unfolded proteins in the endoplasmic reticulum lumen and transmit this stimulation to other areas of the cell. As shown in > Fig. 2 , the expression levels of IRE1 was decreased by MPP + and $100 \mathrm{nM}$ GLP-2 agonist and $100 \mathrm{nM}$ Liraglutide both inhibited the decrease of IRE1 significantly $(P<0.05)$. MPP + has been shown to perturb the autophagy flux [30]. In this light, we investigated the expression of autophagy-related ATC7 and LC3B (II) proteins following GLP-2 agonist or $100 \mathrm{nM}$ Liraglutide pretreatment and MPP + stress. ATG7 is used to assemble autophagosomes and LC3B is a marker of autophagy, the content of which is proportional to the degree of autophagy. As shown in > Fig. 2, 2 mM MPP + increased the expression of both ATG7 and LC3B (II) significantly ( $P<0.05), 100 \mathrm{nM}$ GLP-2 agonist and $100 \mathrm{nM}$ Liraglutide both inhibited the increase of the two protein levels induced by MPP + significantly $(P<0.05)$. $\mathrm{Bcl}-2$ exists in the outer membrane of mitochondria, nuclear membrane and endoplasmic reticulum which has the effect of inhibiting apoptosis. $2 \mathrm{mM} \mathrm{MPP}+$ decreased the expression of $\mathrm{Bcl}-2$ in both cell lines significantly $(P<0.05)$. GLP-2 agonist and $100 \mathrm{nM}$ Liraglutide both inhibited the decrease of $\mathrm{Bcl}-2$ expression induced by MPP + ( Fig. 2$)$ significantly $(P<0.05)$.

\section{GLP-2 agonist protects cells from the increase of mitochondrial superoxide induced by MPP +}

MitoSOX red was used to detect the mitochondrial superoxide in cells. As shown in - Fig 3, mitochondrial superoxide was stained with red which represented positive cells, $2 \mathrm{mM}$ MPP + increased the positive rate and GLP-2 agonist and $100 \mathrm{nM}$ Liraglutide both in- 


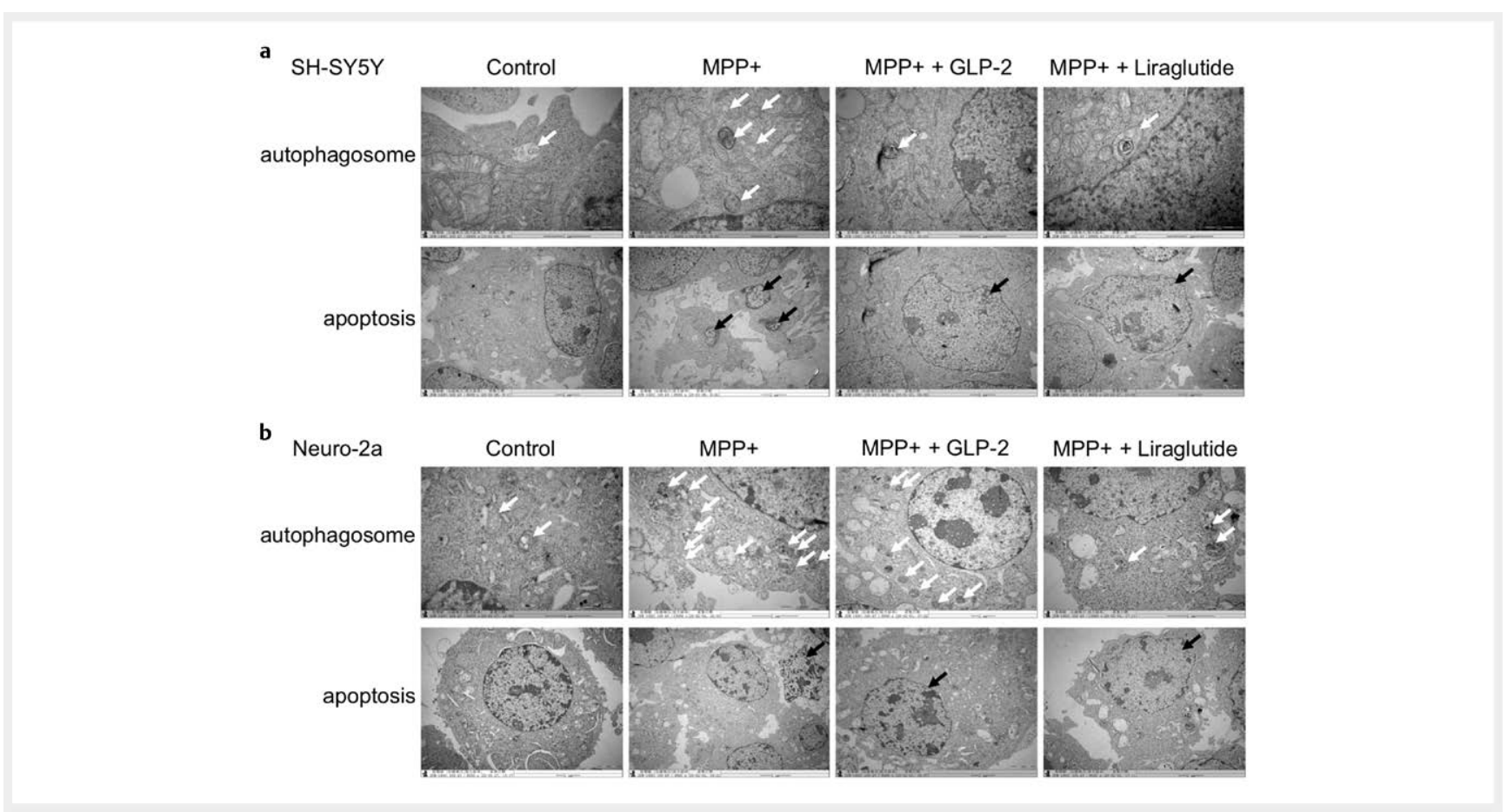

Fig. 4 Mitochondrial morphology, autophagosome and apoptosis using TEM. The white arrow shows the autophagosome, and the black arrow shows apoptosis.

hibited the increase of mitochondrial superoxide significantly $(P<0.05)$.

\section{GLP-2 agonist protects cells against the increases of autophagy and apoptosis from MPP +}

TEM is the "gold standard" for the observation of autophagy. The ultrastructure of autophagy is characterized by vesicular bodies of different sizes wrapped in a bilayer or multilayer membrane, also known as autophagy vesicles. As shown in $\triangleright$ Fig. $\mathbf{4}$, a large number of autophagy vesicles appeared in the cells of MPP +-treated group, with contents visible in the vesicles. The control group showed clear cell structure and normal organelle morphology. In the GLP-2 agonist and $100 \mathrm{nM}$ Liraglutide-pretreated group, multiple autophagosomes could be seen in both SH-SY5Y and N2a cells. Apoptosis was also observed using TEM, as shown in > Fig. 4. In the MPP + group, the cell volume was reduced and the nucleus cleaved into fragments (SH-SY5Y) or had collapsed and shrank (Neuro-2a). In the GLP-2 agonist and $100 \mathrm{nM}$ Liraglutide-pretreated groups, the apoptosis in SH-SY5Y cells we reduced as nucleus collapsed and shrank less, and in Neuro-2a cells they shrank only slightly.

\section{Discussion}

Previous studies demonstrated that GLP-2 has protective effects on neurons. In vitro studies have shown that GLP-2 could protect neurons from excitotoxic damage [14] and reduce glutamate-induced cell death [15]. In In vivo studies, GLP-2 treatment showed some positive effects in the brain. It was reported that the GLP-2 long-acting analogue (teduglutide) can improve obesity-related neuroinflammation and central stress conditions, and reduce the death of nerve cells via apoptosis by reducing inflammation and oxidative stress in the brain [6]. Recent studies reported that GLP-2 can rescue memory impairments and neuropathological changes in mouse models of sporadic $A D$ induced by the intracerebroventricular administration of streptozotocin, this is due to its potential to reduce oxidative stress [5]. Importantly, GLP-2 signaling had clear neuroprotective effects in a re-perfusion stroke model in rats [9] and improved learning, memory and cognitive ability in a cerebral ischemia mouse model of vascular dementia [8]. GLP-2 decreased oxidative damage in the cerebral ischemia/reperfusion model, in which GLP-2 increased the MDA (malondialdehyde) levels and MPO (myeloperoxidase) activity, and reduced the number of apoptotic hippocampal neurons [7]. In addition, the protective effect of GLP-2 on memory impairment in lipopolysaccharide (LPS)treated mice were investigated, and it was found that GLP-2 can reduce the inflammation response and protect and improve memory function [12]. GLP-2 can induce antidepressant-like effects on adrenocorticotropic hormone (ACTH)-treated mice $[10,11]$.

In our study, the viability and cytotoxicity of MPP + on SH-SH5Y and Neuro-2a cells were investigated. MPP + is a toxic radical that is produced in neurons when metabolizing MPTP, which can induce $\mathrm{PD}$ in humans [31]. The experimental results show that the cytotoxicity of MPP + was dose-dependent, and $2 \mathrm{mM} \mathrm{MPP}+$ was selected for subsequent experiments. In the MPP + cell damage model, $100 \mathrm{nM}$ of the GLP-2 agonist or Liraglutide increased cell viability significantly. At the same time, the GLP-2 agonist and Liraglutide both reduced MPP + induced LDH release. Our study demonstrates that the GLP-2 agonist protects neuronal cells through ways of protecting mitochondria, enhancing autophagy, and reducing apoptotic signaling. On the one hand, the GLP-2 analogue 
reversed the MPP + induced decrease of PGC- $1 \alpha$, Mfn2, and IRE1 levels, and decreased mitochondrial superoxide levels, thus reducing oxidative stress in mitochondria and endoplasmic reticulum stress. On the other hand, the GLP-2 agonist normalized autophagy and restored the function of mitochondria, which was also confirmed by electron microscopy. Finally, the GLP-2 analogue inhibited apoptosis by promoting the expression of $\mathrm{BCl}-2$, and TEM images also confirmed that the GLP-2 analogue decreased apoptosis. Our results show that MPP + induced cell damage, activated mitochondrial autophagy and disrupted autophagy homeostasis, leading to mitochondrial oxidative stress injury and mitochondrial dysfunction. Thus, like the GLP- 1 agonist Liraglutide, GLP-2 has protective effect on neuronal cells. As GLP-1 receptor agonists show neuroprotective effects in different animal models and have shown good protective effects in first clinical trials in AD and PD patients $[23,24]$, the observation that the GLP-2 analogue displays comparable neuroprotective effects in this study is of great interest and establishes the GLP-2 signaling pathway as a promising drug target to develop novel treatments for PD.

\section{Conclusion}

In conclusion, GLP-2 agonist protects neuronal cells against mitochondrial damage, autophagy impairments and apoptosis induced by MPP + both in SH-SY5Y and Neuro-2a cells. GLP-2 receptor agonists have the potential to act as a novel treatment for PD, perhaps in unison with GLP-1 analogues.

\section{Conflict of Interest}

The authors declare that they have no conflict of interest.

\section{References}

[1] Suzuki R, Brown GA, Christopher JA et al. Recent developments in therapeutic peptides for the glucagon-like peptide 1 and 2 receptors. J Med Chem 2020; 63: 905-927

[2] Tang-Christensen M, Larsen P], Thulesen J et al. The proglucagonderived peptide, glucagon-like peptide-2, is a neurotransmitter involved in the regulation of food intake. Nat Med 2000; 6: 802-807

[3] Lu Y, Lu D, Hu Y. GLP2 Promotes Directed differentiation from osteosarcoma cells to osteoblasts and inhibits growth of osteosarcoma cells. Molecular Therapy - Nucleic Acids 2018; 10: 292-303

[4] Arda-Pirincci P, Oztay F, Bayrak BB et al. Teduglutide, a glucagon-like peptide 2 analogue: A novel protective agent with anti-apoptotic and anti-oxidant properties in mice with lung injury. Peptides 2012; 38 : 238-247

[5] Sasaki-Hamada S, Ikeda M, Oka J-I. Glucagon-like peptide-2 rescues memory impairments and neuropathological changes in a mouse model of dementia induced by the intracerebroventricular administration of streptozotocin, Sci Rep 2019; doi:10.1038/s41598-019-50167-3

[6] Nuzzo D, Baldassano S, Amato A et al. Glucagon-like peptide-2 reduces the obesity-associated inflammation in the brain. Neurobiol Dis 2019; 121: 296-304

[7] Topaloğlu N, Memi G, Kaner T et al. Does Glp-2 have a protective effect on cerebral ischemia/reperfusion model? Turkish Journal of Medical Sciences 2015; 45: 467-473
[8] Chi CL, Zhang SA, Liu Z et al. Research on the role of GLP-2 in the central nervous system EPK signal transduction pathway of mice with vascular dementia. Eur Rev Med Pharmacol Sci 2017; 21: 131-137

[9] Xie Y-C, Yao Z-H, Yao X-L et al. Glucagon-like peptide-2 receptor is involved in spatial cognitive dysfunction in rats after chronic cerebral hypoperfusion. Journal of Alzheimer's Disease 2018; 66: 1559-1576

[10] Sasaki-Hamada S, Yuri Y, Hoshi M et al. Immunohistochemical determination of the site of antidepressant-like effects of glucagonlike peptide-2 in ACTH-treated mice. Neuroscience 2015; 294 : 156-165

[11] Sasaki-Hamada S, Nakamura R, Nakao Y et al. Antidepressant-like effects exerted by the intranasal administration of a glucagon-like peptide- 2 derivative containing cell-penetrating peptides and a penetration-accelerating sequence in mice. Peptides 2017; 87: 64-70

[12] Iwai T, Jin K, Ohnuki T et al. Glucagon-like peptide-2-induced memory improvement and anxiolytic effects in mice. Neuropeptides 2015; 49: 7-14

[13] Amato A, Baldassano S, Mulè F. GLP2: an underestimated signal for improving glycaemic control and insulin sensitivity. J Endocrinol 2016; 229: R57-R66

[14] Vrang N, Larsen PJ. Preproglucagon derived peptides GLP-1, GLP-2 and oxyntomodulin in the CNS: Role of peripherally secreted and centrally produced peptides. Progress in Neurobiology 2010; 92: 442-462

[15] Lovshin JA, Huang Q, Seaberg R et al. Extrahypothalamic expression of the glucagon-like peptide-2 receptor is coupled to reduction of glutamate-induced cell death in cultured hippocampal cells. Endocrinology 2004; 145: 3495-3506

[16] Drucker DJ, Yusta B. Physiology and pharmacology of the enteroendocrine hormone glucagon-like peptide-2. Annu Rev Physiol 2014; 76: 561-583

[17] El-Jamal N, Erdual E, Neunlist M et al. Glugacon-like peptide-2: broad receptor expression, limited therapeutic effect on intestinal inflammation and novel role in liver regeneration. Am J Physiol Gastrointest Liver Physiol 2014; 307: G274-G285

[18] Guan X. The CNS glucagon-like peptide-2 receptor in the control of energy balance and glucose homeostasis. Am J Physiol Regul Integr Comp Physiol 2014; 307: R585-R596

[19] Wang Y, Guan X. GLP-2 potentiates L-type Ca 2+ channel activity associated with stimulated glucose uptake in hippocampal neurons. Am J Physiol Endocrinol Metab 2010; 298: E156-E166

[20] Shi X, Li X, Wang Y et al. Glucagon-like peptide-2-stimulated protein synthesis through the PI 3-kinase-dependent Akt-mTOR signaling pathway. Am J Physiol Endocrinol Metab 2011; 300: E554-E563

[21] Liu W, Jalewa J, Sharma $M$ et al. Neuroprotective effects of lixisenatide and liraglutide in the 1-methyl-4-phenyl-1,2,3,6-tetrahydropyridine mouse model of Parkinson's disease. Neuroscience 2015; 303: 42-50

[22] Zhang L, Zhang L, Li Y et al. The Novel Dual GLP-1/GIP Receptor Agonist DA-CH5 Is Superior to Single GLP-1 Receptor Agonists in the MPTP Model of Parkinson's Disease. Journal of Parkinson's Disease 2020; $1-20$

[23] Hölscher C. Novel dual GLP-1/GIP receptor agonists show neuroprotective effects in Alzheimer's and Parkinson's disease models. Neuropharmacology 2018; 136: 251-259

[24] Athauda D, Maclagan K, Skene SS et al. Exenatide once weekly versus placebo in Parkinson's disease: a randomised, double-blind, placebo-controlled trial. The Lancet 2017; 390: 1664-1675

[25] Lund A, Vilsbøll T, Bagger Jl et al. The separate and combined impact of the intestinal hormones, GIP, GLP-1, and GLP-2, on glucagon secretion in type 2 diabetes. Am J Physiol Endocrinol Metab 2011; 300: E1038-E1046

[26] Sharma MK, Jalewa J, Hölscher C. Neuroprotective and anti-apoptotic effects of liraglutide on SH-SY5Y cells exposed to methylglyoxal stress. J Neurochem 2014; 128: 459-471 
[27] Jalewa J, Sharma MK, Hölscher C. Novel incretin analogues improve autophagy and protect from mitochondrial stress induced by rotenone in SH-SY5Y cells. J Neurochem 2016; 139: 55-67

[28] Panagaki T, Michael M, Hölscher C. Liraglutide restores chronic ER stress, autophagy impairments and apoptotic signalling in SH-SY5Y cells. Sci Rep 2017; 7: 16158

[29] Ye Q, Chen C, Si E et al. Mitochondrial Effects of PGC-1alpha Silencing in MPP + Treated Human SH-SY5Y Neuroblastoma Cells. Frontiers in Molecular Neuroscience 2017; 10
[30] Janda E, Isidoro C, Carresi C et al. Defective Autophagy in Parkinson's Disease: Role of Oxidative Stress. Molecular Neurobiology 2012; 46: 639-661

[31] Gerlach M, Riederer P, Przuntek $H$ et al. MPTP mechanisms of neurotoxicity and their implications for Parkinson's disease. European Journal of Pharmacology 1991; 208: 273-286 